Canad. Math. Bull. Vol. 21 (4), 1978

\title{
AN ASYMPTOTIC FORMULA FOR RECIPROCALS OF LOGARITHMS OF CERTAIN MULTIPLICATIVE FUNCTIONS
}

\author{
BY \\ JEAN-MARIE DE KONINCK AND ALEKSANDAR IVIĆ
}

Sums of the form $\sum_{n \leq x}^{\prime} 1 / \log f(n)$, where $f(n)$ is a multiplicative arithmetical function and $\Sigma^{\prime}$ denotes summation over those values of $n$ for which $f(n)>0$ and $f(n) \neq 1$, were studied by De Koninck [2], De Koninck and Galambos [3], Brinitzer [1] and Ivić [5]. The aim of this note is to give an asymptotic formula for $\sum_{n \leq x}^{\prime} 1 / \log f(n)$ for a certain class of multiplicative, positive, primeindependent functions (an arithmetical function is prime-independent if $f\left(p^{\nu}\right)=$ $g(\nu)$ for all primes $p$ and $\nu=1,2, \ldots)$. This class of functions includes, among others, the functions $a(n)$ and $\tau^{(e)}(n)$, which represent the number of nonisomorphic abelian groups of order $n$ and the number of exponential divisors of $n$ respectively, and none of the estimates of the above-mentioned papers may be applied to this class of functions. We prove the following.

THEOREM. Let $f(n)$ be a multiplicative arithmetical function such that for all primes $p$ and $\nu=1,2, \ldots$ we have $f\left(p^{\nu}\right)=g(\nu)$, where $g(1)=1, g(\nu)>1$ for $\nu \geq 2$ and $\lim \inf _{\nu \rightarrow \infty} g(\nu)>1$. Then we have

$$
\sum^{\prime} 1 / \log f(n)=x \int_{-\infty}^{0}\left(C(t)-6 / \pi^{2}\right) d t+0\left(x^{1 / 2} \log ^{1 / 2} x\right),
$$

where $C(t)=\Pi_{p}\left(1+\sum_{k=2}^{\infty}\left(g^{t}(k)-g^{t}(k-1)\right) p^{-k}\right)$, and $\sum^{\prime}$ denotes summation over those values of $n$ for which $f(n)>1$.

Proof. First of all $f(n) \geq 1$, and $f(n)=1$ if and only if $n$ is square-free, or equivalently if and only if $1-\mu^{2}(n)=0$, where $\mu(n)$ is the Möbius function.

Let us define

$$
\sum_{n \leq x}^{\prime} f^{t}(n)=\sum_{n \leq x, f(n)>1} f^{t}(n)
$$

Then we have

$$
\text { (3) } \begin{aligned}
\sum_{n \leq x}^{\prime} f^{t}(n)=\sum_{n \leq x}(1- & \left.\mu^{2}(n)\right) f^{t}(n)=\sum_{n \leq x} f^{t}(n)-\sum_{n \leq x} \mu^{2}(n) \\
& =\sum_{n \leq x} f^{t}(n)-\frac{6}{\pi^{2}} x+0\left(x^{1 / 2} \exp \left(-C \log ^{3 / 5} x(\log \log x)^{-1 / 5}\right)\right),
\end{aligned}
$$

where $C$ is a positive constant (see [9]).

Received by the editors September 19, 1977 and, in revised form, February 6, 1978. 
We now proceed to estimate $\sum_{n \leq x} f^{t}(n)$ for $t \leq 0$. For $\operatorname{Re} s>1$ we clearly have

$$
\begin{aligned}
\sum_{n=1}^{\infty} f^{t}(n) n^{-s}=\prod_{p}\left(1+p^{-s}+g^{t}(2) p^{-2 s}+g^{t}(3) p^{-3 s}+\cdots\right) \\
=\zeta(s) \prod_{p}\left(1+\sum_{k=2}^{\infty}\left(g^{t}(k)-g^{t}(k-1)\right) p^{-k s}\right)=\zeta(s) g(s, t),
\end{aligned}
$$

where $g(s, t)=\sum_{n=1}^{\infty} h(n, t) n^{-s}, h(n)$ is multiplicative and

$$
h\left(p^{j}, t\right)=\left\{\begin{array}{cc}
0 & j=1 \\
g^{t}(j)-g^{t}(j-1) & j \geq 2 .
\end{array}\right.
$$

Since $t \leq 0$ we have $|h(n, t)| \leq u(n)$, where

$$
u(n)=\left\{\begin{array}{lc}
0 & \text { if there is a } p \text { such that } p \| n, \\
1 & \text { otherwise }
\end{array}\right.
$$

so that $\sum_{n \leq x}|h(n, t)| \leq \sum_{n \leq x} u(n)=0\left(x^{1 / 2}\right)$. Denoting $w=g^{t}(2)$ for shortness, further factoring yields

$$
g(s, t)=\zeta^{w-1}(2 s) u(s, t)
$$

where for $t \leq 0$,

$$
\begin{aligned}
u(s, t) & =\prod_{p}\left(1-p^{-2 s}\right)^{w-1}\left(1+(w-1) p^{-2 s}+\left(g^{t}(3)-w\right) p^{-3 s}+\cdots\right) \\
& =\prod_{p}\left(1+\left(g^{t}(3)-w\right)\left(1-p^{-2 s}\right)^{w-1} p^{-3 s}+0\left(p^{-4 s}\right)\right),
\end{aligned}
$$

so that if $\sum_{n=1}^{\infty} c(n, t) n^{-s}=u(s, t)$, then for every $\varepsilon>0$ and uniformly in $t \leq 0$,

$$
\sum_{n \leq x}|c(n, t)|=0\left(x^{1 / 3+\varepsilon}\right)
$$

and partial summation gives

$$
\sum_{n>x}|c(n, t)| n^{-1 / 2}=0\left(x^{-1 / 6+\varepsilon}\right) .
$$

If we set $\sum_{n=1}^{\infty} b(n, t) n^{-s}=\zeta^{w-1}(2 s)$, then we have by a result of A. Selberg [7]

$$
\sum_{n \leq x} b(n, t)=\Gamma^{-1}(w-1) 2^{2-w} x^{1 / 2} \log ^{w-2} x+0\left(x^{1 / 2} \log ^{w-3} x\right)
$$

which gives uniformly in $t \leq 0$

$$
\sum_{n \leq x} b(n, t)=0\left(x^{1 / 2} \log ^{w-2} 2 x\right) .
$$


From (4) it follows that

$$
\sum_{n \leq x} h(n, t)=\sum_{n \leq x} c(n, t) \sum_{m \leq x / n} b(m, t)=0\left(x^{1 / 2} \sum_{n \leq x}|c(n, t)| n^{-1 / 2} \log ^{w-2} 2 x / n\right) .
$$

Since $\sum_{n=1}^{\infty}|c(n, t)| n^{-1 / 2}$ converges we have

$$
\begin{aligned}
& \sum_{n \leq x}|c(n, t)| n^{-1 / 2} \log ^{w-2} 2 x / n=\sum_{n \leq x}+\sum_{x^{1 / 2}<n \leq x} \\
& =0\left(\log ^{w-2} x\right)+0\left(\sum_{n>x^{1 / 2}}|c(n, t)| n^{-1 / 2}\right)=0\left(\log ^{w-2} x\right)+0\left(x^{-1 / 12+\varepsilon}\right)=0\left(\log ^{w-2} x\right)
\end{aligned}
$$

so that

$$
\sum_{n \leq x} h(n, t)=0\left(x^{1 / 2} \log ^{w-2} x\right)=0\left(x^{1 / 2} \log ^{-1} x\right),
$$

since for $t \leq 0$ we have $w \leq 1$, and partial summation gives

$$
\sum_{n>x} h(n, t) n^{-1}=0\left(x^{-1 / 2} \log ^{-1} x\right) .
$$

Now take $y=x / \log x, z=\log x$. From $\sum_{n=1}^{\infty} f^{t}(n) n^{-s}=\zeta(s) g(s, t)$ we get

$$
\begin{aligned}
\sum_{n \leq x} f^{t}(n)=\sum_{m n \leq x} h(n, t)= & \sum_{n \leq y} h(n, t)[x / n]+\sum_{m \leq z} 1 \sum_{n \leq x / m} h(n, t) \\
& -\sum_{m \leq z} 1 \sum_{n \leq y} h(n, t)=S_{1}+S_{2}-S_{3} .
\end{aligned}
$$

$S_{3}=0\left(z y^{1 / 2} \log ^{-1} x\right)=0\left(x^{1 / 2} \log ^{-1 / 2} x\right)$.

$S_{2}=0\left(x^{1 / 2} \sum_{m \leq z} m^{-1 / 2} \log ^{-1} x / m\right)=0\left(x^{1 / 2} \log ^{-1} y \sum_{m \leq z} m^{-1 / 2}\right)=0\left(x^{1 / 2} \log ^{-1 / 2} x\right)$.

$S_{1}=\sum_{n \leq y} h(n, t)(x / n+0(1))=C(t) x+x \sum_{n>y} h(n, t) n^{-1}+0\left(\sum_{n \leq y}|h(n, t)|\right)$

$$
=C(t) x+0\left(x y^{-1 / 2} \log ^{-1} y\right)+0\left(y^{1 / 2}\right)=C(t) x+0\left(x^{1 / 2} \log ^{-1 / 2} x\right),
$$

so that we obtain uniformly in $t$

$$
\sum_{n \leq x} f^{t}(n)=C(t) x+0\left(x^{1 / 2} \log ^{-1 / 2} x\right)
$$

where

$$
C(t)=g(1, t)=\sum_{n=1}^{\infty} h(n, t) n^{-1}=\prod_{p}\left(1+\sum_{k=2}^{\infty}\left(g^{t}(k)-g^{t}(k-1)\right) p^{-k}\right) .
$$


Putting (7) into (3) and integrating from $-T$ to $0(T>0)$ we get

(8) $\sum_{n \leq x}^{\prime} 1 / \log f(n)=x \int_{-T}^{0}\left(C(t)-6 / \pi^{2}\right) d t+0\left(x^{1 / 2} \log ^{-1 / 2} x \cdot T\right)$

$$
+0\left(T x^{1 / 2} \exp \left(-C \log ^{3 / 5} x(\log \log x)^{-1 / 5}\right)\right)+\sum_{n \leq x} f^{-T}(n) / \log f(n) .
$$

To estimate $C(t)-6 / \pi^{2}$ for $t \leq 0$, let $C(t)=\Pi_{p}\left(1-p^{2}+u(p, t)\right)$, where

$$
\begin{aligned}
0<u(p, t) & =g^{t}(2) p^{-2}+\left(g^{t}(3)-g^{t}(2)\right) p^{-3}+\left(g^{t}(4)-g^{t}(3)\right) p^{-4}+\cdots \\
& =g^{t}(2)\left(p^{-2}-p^{-3}\right)+g^{t}(3)\left(p^{-3}-p^{-4}\right)+g^{t}(4)\left(p^{-4}-p^{-5}\right)+\cdots \\
& \leq g^{t}(r)\left(p^{-2}-p^{-3}+p^{-3}-p^{-4}+p^{-4}-\cdots\right)=g^{t}(r) p^{-2}
\end{aligned}
$$

where $r$ is an integer such that $g(\nu) \geq g(r)>1$ for $\nu=2,3, \ldots$ Such an integer certainly exists, since $\lim \inf _{\nu \rightarrow \infty} g(\nu)>1$.

Using the inequality $\log (x+y) \leq \log x+y / x(x, y>0)$ we get

$$
\begin{aligned}
C(t) & =\exp \left(\log \prod_{p}\left(1-p^{-2}+u(p, t)\right)=\exp \left(\sum_{p} \log \left(1-p^{-2}+u(p, t)\right)\right.\right. \\
& \leq \exp \left(\sum_{p} \log \left(1-p^{-2}\right)+\sum_{p}\left(1-p^{-2}\right)^{-1} u(p, t)\right) \\
& \leq \frac{6}{\pi^{2}} \exp \left(g^{t}(r) \sum_{p}\left(p^{2}-1\right)^{-1}\right) \leq 6 \exp \left(g^{t}(r)\right) / \pi^{2} .
\end{aligned}
$$

If $t<0$ is small enough we get

$$
\begin{aligned}
& 0 \leq C(t)-6 / \pi^{2} \leq\left(6 / \pi^{2}\right)\left(\exp \left(g^{t}(r)\right)-1\right)=0\left(g^{t}(r)\right), \\
& \int_{-\infty}^{-T}\left(C(t)-6 / \pi^{2}\right) d t=0\left(\int_{-\infty}^{-T} g^{t}(r) d t\right)=0\left(g^{-T}(r)\right) .
\end{aligned}
$$

If $n=p_{1}^{\nu_{1}} \cdots p_{i}^{\nu_{i}}$, then $f(n)=g\left(\nu_{1}\right) \cdots g\left(\nu_{i}\right) \geq g(r)>1$ if $f(n)>1$, so that $f^{T}(n) \log f(n) \geq g^{T}(r) \log g(r)$ if $f(n)>1$, and we obtain

(11) $\sum_{n \leq x}^{\prime} f^{-T}(n) / \log f(n) \leq \sum_{n \leq x}^{\prime} g^{-T}(r) / \log g(r)=0\left(g^{-T}(r) \sum_{n \leq x} 1\right)=0\left(g^{-T}(r) x\right)$.

Writing $\int_{-T}^{0}\left(C(t)-6 / \pi^{2}\right) d t=\int_{-\infty}^{0}-\int_{-\infty}^{-T}$ and using (10) and (11) we get from (8)

$$
\begin{aligned}
\sum_{n \leq x}^{\prime} 1 / \log f(n)=x \int_{-\infty}^{0}\left(C(t)-6 / \pi^{2}\right) d t & +0\left(g^{-T}(r) x\right)+0\left(x^{1 / 2} \log ^{-1 / 2} x . T\right) \\
& +0\left(T x^{1 / 2} \exp \left(-C \log ^{3 / 5} x(\log \log x)^{-1 / 5}\right)\right) .
\end{aligned}
$$

Now take $T=\log x / 2 \log g(r)$. Then we have

$$
g^{-T}(r) x=\exp (-T \log g(r)+\log x)=\exp \left(\frac{1}{2} \log x\right)=x^{1 / 2},
$$

and so the theorem is proved. 
As a first example, let us take $a(n)$, the number of non-isomorphic abelian groups of order $n$. It is well-known (see [4]) that $a(n)$ is multiplicative, and that $a\left(p^{\nu}\right)=P(\nu)$ for any prime $p$ and $\nu=1,2, \ldots$, where $P(\nu)$ is the number of unrestricted partitions of the integers $\nu$, so that $P(\nu)=1$ if $\nu=1$ and $P(\nu)$ is strictly increasing with $\nu$. Therefore the conditions of our theorem are satisfied, and (1) holds with $f(n)=a(n), g(k)=P(k)$. Note that in this case we have $\liminf _{\nu \rightarrow \infty} g(\nu)=+\infty$ and $r=2, g(r)=2$.

Examples of other multiplicative, prime-independent functions that satisfy the conditions of our theorem may be readily found among enumerative functions of certain algebraic structures. Such is for example (see [6] for a detailed discussion) $S(n)$, the number of non-isomorphic semisimple finite rings of order $n$.

Finally let us consider $\tau^{(e)}(n)$, the number of exponential divisors of $n$. A divisor $d=p_{1}^{b_{1}} \cdots p_{i}^{b_{i}}$ is called an exponential divisor of $n=p_{1}^{\nu_{1}} \cdots p_{i}^{\nu_{i}}$ if $b_{1}\left|\nu_{1}, \ldots, b_{i}\right| \nu_{i}$ (see [8]). It follows that $\tau^{(e)}(n)$ is a multiplicative, primeindependent arithmetical function for which $\tau^{(e)}\left(p^{\nu}\right)=\tau(\nu)$, where $\tau(\nu)$ is the ordinary number of divisors function. Since $\tau(1)=1$ and $\tau(\nu) \geq 2$ if $\nu \geq 2$, the conditions of our theorem are satisfied and (1) holds with $f(n)=\tau^{(e)}(n)$ and $g(k)=\tau(k)$. Again it is of interest to note that $\liminf _{\nu \rightarrow \infty} g(\nu)=2$ and $r=2$, $g(r)=2$ also.

\title{
REFERENCES
}

1. E. Brinitzer, Eine asymptotische Formel für Summen über die reziproken Werte additiver Funktionen, Acta Arith. XXXII, 1977, pp. 387-391.

2. J.-M. De Koninck, On a class of arithmetical functions, Duke Math. Journal, 39, 1972, pp. 8ग7-818.

3. J.-M. De Koninck and J. Galambos, Sums of reciprocals of additive functions, Acta Arith. XXV, 1974, pp. 159-164.

4. I. N. Herstein, Topics in Algebra, Blaisdell, Waltham, Mass.-Toronto-London, 1964.

5. A. Ivić, The distribution of values of some multiplicative functions, Publications de l'Institut Math. (Belgrade), 22 (36), 1977, pp. 87-94.

6. J. Knopfmacher, Abstract Analytic Number Theory, North-Holland/American Elsevier, Amsterdam-Oxford, 1975.

7. A. Selberg, Note on a paper by L. G. Sathe, J. Indian Math. Soc., 18, 1954, pp. 83-87.

8. M. V. Subbarao, On some arithmetic convolutions in the theory of arithmetic functions, Lecture Notes in Math. 251, Springer-Verlag, Berlin-Heidelberg-New York, 1972, pp. 247-271.

9. A. Walfisz, Weylsche Exponentialsummen in der neueren Zahlentheorie, VEB Verlag, Berlin, 1963, pp. 192-198.

\author{
JeAn-Marie De Koninck \\ DÉPARTMENT de MATHÉMATIQUES \\ UNIVERSITÉ LAVAL \\ QuÉbec, P.Q. CANADA, G1K 7P4
}

\author{
Aleksandar Ivić \\ RUDARSKO-GEOLOŠKI FAKULTET \\ DJUŠINA 7, 11000 BEOGRAD \\ YugosLavia
}

